\title{
Religiosity Orientations and Personality Traits with Death Obsession
}

\author{
Hamzeh Salmanpour (Corresponding author) \\ Faculty of Psychology, AZAD University of Naqadeh, Naqadeh, Iran \\ E-mail: salmanpour_hamzeh@yahoo.com
}

Ali Issazadegan

Faculty of Psychology, The University of Urmia, Urmia, Iran

E-mail: ali_issazadeg@yahoo.com

Received: December 17, 2011

doi:10.5539/ijps.v4n1p150
Accepted: January 10, $2012 \quad$ Published: March 1, 2012

URL: http://dx.doi.org/10.5539/ijps.v4n1p150

\begin{abstract}
The aim of the current study was to investigate the relationship and predictability of death obsession through religiosity orientations and personality traits. Sample included 484 (246 girls, 238boys) that had been chosen through random stratified sampling. In order to assess research instrument was death obsession scale (DOS), NEO personality inventory (NEO-FFI) and Allport religious orientation scale. Data were analyzed using correlation and stepwise regression analyses method and t-test.

Results showed that the relationship between death obsessions with extrinsic orientation toward religion is positive whereas death obsession has a negative relation with intrinsic orientation toward religion. Also findings showed there is significant positive relationship between neuroticism and death obsession $(r=0 / 42, p<0 / 01)$. Other dimensions at personality had negative relationship with death obsession. The greater negative relationship was between intrinsic orientation toward religion and conscientiousness dimension $(\mathrm{r}=-0 / 34, \mathrm{p}<0 / 01)$. Of all research variables, extrinsic orientation toward religion and neuroticism was able to predict 19 present of variance of death obsession. In study of difference between two group female and male in death obsession results showed that significant difference between two groups $(\mathrm{t}=5 / 38, \mathrm{df}=482, \mathrm{p}<0 / 001)$.
\end{abstract}

Keywords: Death obsession, Religiosity orientation, Personality traits

\section{Introduction}

The history of human thinking has directed itself to death and life from the beginning. Every living creature would finally die. But among all these creatures it's just human who is aware of this truth. Thinking about death always has been a terrifying issue and many prefer not to meditate about it. Ibn Sina known as Avec cina (980-1037) has considered the truth of death and dread of it in his epistle which name is "ash shifa (Healing)". He has considered the fear of death as the greatest fear of human. There is no doubt that death and afterlife and human's view of it is one of the substantial issues that have been noticed by all of the religions.

Speaking about death leads to creation of excitements as fear, sadness and indignation. In such a manner that severs and intensified excitements as depression is considered as the fourth stage of dying (Abdel-khalek, 1997). Getting aware of mortality and perishableness of human life, death related thoughts and anxiety that is caused by it leads to regularizing the reactions of human with the others (Semeichel, Gailliot, Filardo, McGregor, \& Gitter, 2009). Based on theory of terror management (Rossenblat, Greenberg, Solomon, Pyszczynski \& Lyon, 1989) recalling mortality, by causing agitation in individuals provoke them to decrease it and puts impression on individual's social cognition.

Templar et al. (1993) described that besides ordinary attitude toward death, three hypothetical and imaginary approaches have been mooted in relation to death distress. That has been called as death depression, death anxiety and death obsessions. The two elements of death depression and death anxiety already have been propounded and have been surveyed by researchers. 
The third component in this area, namely death depression has been introduced by Abdel-khalek (1994) he holds this theory that there would be possibly a relationship or overlapping between death and obsessions. A study about obsession symptoms indicates that more than $70 \%$ of obsessive death-rated thoughts are one of the commonest and the most prevailing experiences among obsessive patients. Multby and Day (2000) have reported correlation coefficient of $(\mathrm{r}=0 / 51)$ between common obsession and death obsession.

The obsessions are regarded as mental images, unintentional impulses or intruders that occur without tendency and intention of individual and experienced incompatibly and illegibly with self. The individuals resist activity against obsessions and know that they are not the productions of his mind. About death and obsession the insisting idea of the person on death is of high importance (Rajabi, 2009).

Death obsession refers to impulses, concerns and death related believes. In connection with this 3 factors of death rumination, death dominance and repetitive views or beliefs directed to death have been pointed out (Abdel-khalek, 1998). The researches have shown that in connection to attitudes and reactions to the death phenomenon and death in natural way or neuroticism there can be individual differences. We can point to attitude and religiosity orientation and character. Religion as a fundamental motivation in individual's life has very special status. And introduces a meaningful network for a faithful person who in its context the whole life is understood (Watson \& Ghorbani, 2000). Most of the studies on this issue have reported a negative relationship between faithfulness (religiosity) and death anxiety (Duff, Lawrence \& Hong, 1995). Lunette and Templar (1986) have reported that the studies indicate religious meditations, Personal religious activities like saying prayer, worshipping and reading sacred texts, value of relationship with friends and family members, value of participation of individuals in group works, gender, age, marital status, level of income and healthy mood have their impacts on death anxiety. One of the variables which can have a relationship with obsessive view toward death is religiosity orientation in its connection Allport's view about intrinsic and extrinsic orientation toward religion can be mooted. Intrinsic religious orientation is experiencing religion as an important motivational factor which is completely has been internalized and practical in individual's life. And extrinsic religious orientation which is equivalent to undeveloped religion includes religiosity and religion use to obtain some goals like social support. Several studies in Iran and other countries, indicates that there is a positive and meaningful relationship between religiosity and mental health.

Also, a negative and meaningful relationship between religiosity orientation and Death depression and anxiety has been reported.34 percent out of 130 researches which were done indicate positive and meaningful impact of religiosity on depression and anxiety. Due to specific style of life that people with intrinsic religious orientation which they have, they regard and take stress as an opportunity to improve their spiritual and virtual experiences (Hamid, 2009). Or regard it as a god's destiny. Some scholars hold this view that religion to some extant, provides individuals with personality integration. That how religiosity orientation effects mental health of individuals has been studies many times by scholars (Compton, 2005). The researches have brought forward the religiosity orientation with personality components. Studies in connection to the relationship between personality and religiosity orientation indicate that there is a relationship between religiosity and some characteristics of the individuals. Eysenk (1967-1975; as cited in Maltby, 1999) believes that there is a relationship between flexibility and religious attitudes.

Jorm and Christensen (1992) believed that there is a negative relationship between religiosity and psychotism. The research has also reported that there is a negative relationship between being psychosis and religiosity orientation especially in intrinsic one (Egan, Kroll, Carey, Johnson, Erickson, 2004). Other studies have shown the correlation and relationship between religiosity and extroversion, the $\mathrm{G}$ factor in cattle theory with religiosity orientation (Francis and Pearson, 2001; Francis \& Bourke, 2003). The studies on five factorial pattern of personality have also shown that intrinsic religiosity orientation is related to agreeableness, conscientiousness and extroverts and extrinsic religiosity orientation has specifically a positive relationship with neuroticism (Heningsgard \& Arnau, 2008).

Saroglou (2002) in a Meta- analytical study found that there is a relationship between personality and religiosity orientation. A positive relationship is there between religisity with extraversion, agreeableness, conscientiousness and openness to experience and being a negative relation between neuroticism and death obsession.the findings showed that among big-five factors of personality, the agreeableness and conscientiousness has the most reliability and most lasting relation with religiosity. They have also shown that there is a relationship between emotional stability and religiosity orientation. The more religiosity orientation gets intrinsic the more will be emotional stability.

Maltby and Day (2000) in surveying the relationship between death obsession and its repercussion point at the 
religiosity orientation and have reported a negative correlation $(\mathrm{r}=-0 / 35)$ and a positive relationship between death obsession and extrinsic religiosity orientation $(\mathrm{r}=0 / 28)$. They have shown that intrinsic religiosity orientation accompanies with low death distress and extrinsic religiosity orientation accompanies with higher death distress. In Abdel-Khalek studies (2002) with surveying death obsession between normal people, patients with anxiety disorder, schizophernia, substance abuse they reached to this conclusion that anxious patients got higher scores than other groups.

Abdel-khalek et al. (2006) showed that the death obsession among women and Palestine men who live in a stressful situation of camps have greater death obsession scale than Spanish, English and American women and men.findings showed that death obsession has a positive relationship with extrinsic religiosity orientation while has a negative relationship with intrinsic religiosity orientation. The findings showed intrinsic religiosity orientation has less relationship with death anxiety in connection to gender and sex. The findings show that the relationship between death obsession and extrinsic religiosity orientation in female group was stronger than a male group (Abdel-khalek, 1998). In studying the relationship of religiosity, death obsession and death anxiety among female nursery students of Egypt at the age range of 17-25, there was a negative significant relation (Al-sabwah \& Abdel-kalek, 2006).

In other study Abdel-khalek and Lester (2003) in studying death obsession among Kuwaiti and American samples showed that women have greater scores than men in death obsession scale. Abdel-khalek (2007) in connection to the relationship of love to life and death obsession found that women have greater average scores than men in three scales of (death distress, death anxiety and death obsession).

With respect to theoretical and research basics of the above mentioned study consequently, the following hypotheses are suggested:

1) There is a relationship between religiosity orientation and death obsession.

2) There is a relationship between death obsession and personality.

3) There is a relationship between personality and religiosity orientation.

4) Intrinsic and extrinsic religiosity orientation and personality have predicted power for death obsession.

5) Between male and female students the death obsession is different.

\section{Method}

\subsection{Subjects}

The population of current research consists of all undergraduate female and male students of Peyam Noor and Azad University of Naqadeh in Iran who enrolled in academic year 2010/2011. The samples for the study were randomly selected from population. The selected sample was 484 students. The participants in the sample had a mean age of $25.15(\mathrm{SD}=6.3)$.

\subsection{Materials}

Death obsession scale (DOS, Abdel-khalek, 1998): includes 15 items which have been made to measure the mental concerns impulses and assess the situations based on 5 items scale. In this scale, $1=$ Never, and $5=$ Very much (greatest). The scores range was between 15-75. DOS has three agents including death rumination, death dominance and repeated believes toward death. Abdel-khalek (1998) in major culture has reports internal consistency and its agents with 0.90 . Reliability of death obsession scale has been reported throng DOS coefficient with death anxiety scale 0.62 , death depression 0.57 , general obsession 0.46 , general anxiety 0.33 and general depression 0.42 . In Iran, researchers have reported the simultaneous validity and reliability of this scale with simultaneous applying of it with death anxiety scale (0.76).

Allport religiosity orientation questionnaire (Allport \&Ross, 1967): includes 20 items which measures the intrinsic and extrinsic religiosity orientation. This scale has two sub-scales, 11 statements relates to extrinsic religiosity orientation and 9 scales to intrinsic religiosity,with response on a 5-point likert scale form.In this scale, the options have been regulated based on discrete scale. For extrinsic 1 (entirely disagree) to 5 (completely agree) and for intrinsic religiosity orientation, 1 (completely agree) to 5 (completely disagree). The reliability of this questionnaire has been surveyed in Iranin sample and Cronbach alpha (0.73) has been reported.

The NEO Five-Factor Inventory(NEO-FFI,Costa\&McCrae,1992) is a personality test which is based on the five-factor model of personality :Extraversion, Openness, Agreeableness, Conscientiousness, Neuroticism .The response of strongly disagree gets a 0 and as we move from disagree to strongly agree, the respective response gets one of the scores from 1 to 4 in the increasing order. NEO-FFI has 60 items in the form of statements. Each 
statement can be responded on a likert scale on which responses range from strongly disagree to strongly agree . The test is not timed and usually takes $15-20$ minutes to compete. There are 12 items for each factor. The internal consistency of the NEO-FFI as calculated using coefficient alpha was 0.86 for Neuroticism, 0.77 for Extraversion, 0.73 for Openness, 0.68 for Agreeableness and 0.81 for Conscintiousness. NEO-FFI is a shorter from of NEO PI-R scale (Costa \&McCrae,1992).The correlation between NEO PI-R various studies have already shown validity and reliability of NEO-FFI and the domain scales of NEO-PI-R were 0.92 for Neuroticism. 0.90 for Extraversion, 0.91 for openness, 0.77 for agreeableness and 0.87 for conscientiousness.

\subsection{Procdure}

The questionnaires were administered anonymously to students during group testing sessions in their classrooms during the academic years 2010/2011.A description of procedure was read to participants by the experimenter. Participants were told that their participation was voluntary.participants was asked to complete a set of questionnaires after giving informed consent.spss. 18 was used for the statistical analyses.

\section{Results}

To study the relationship between death obsession with religiosity orientation and personality traits, we used correlation method. The results show that there is significantly correlation between death obsession and extrinsic religiosity orientation $(\mathrm{r}=0.12, \mathrm{p}<0 / 01)$. The results also showed that besides being open to experience all dimensions of personality trait has meaningful correlation with death obsession which hypothesis (1), (2) of the study are approved. The results also showed that among personality traits except agreeableness, neuroticism and conscientiousness, openness to experience other dimension of personality had significantly correlation with extrinsic religiosity orientation. Extraversion $(\mathrm{r}=-0.23, \mathrm{p}<0 / 01)$, agreeableness $(\mathrm{r}=-0.16, \mathrm{p}<0.01)$, conscientiousness $(\mathrm{r}=-0.34, \mathrm{p}<0.01)$ and openness to experience with $(\mathrm{r}=-0.18, \mathrm{p}<0.01)$ had significantly negative correlation with intrinsic religiosity orientation and extra version with $(r=0.09, p<0.05)$ had significantly correlation with intrinsic religiosity orientation. The highest amount of negative correlation with $(\mathrm{r}=$ $0.34, \mathrm{p}<0.001)$ was seen between intrinsic religiosity orientation with conscientiousness. The finding approves the (1) and (2) and (3) hypothesis of the study.See table 1 for these results.

To determine the role of religiosity orientations and personality traits in predicting death obsession we used step by step regression analysis method. In the first move, the neuroticism variable was entered regression model, the results of regression analysis showed that the effect of neuroticism variable on death obsession was positive and meaningful $(\mathrm{R}=0.42, \mathrm{P}<0.001, \mathrm{df}=1, \mathrm{~F}=105.55)$. So the neuroticism among personality traits can predict death obsession and this variable explains the 18 percent of death obsession variance $\left(\mathrm{R}^{2}=0.18\right)$. On the second step the extrinsic religiosity orientation variable was taken in to regression model and the results of multi regression variance analysis showed that the effect of extrinsic religiosity orientation on death obsession was meaningful and positive $(\mathrm{R}=0.43, \mathrm{P}<0.001, \mathrm{df}=2, \mathrm{~F}=56.58)$. So the extrinsic religiosity orientation can predict death obsession and this variable explains $19 \%\left(\mathrm{R}^{2}=0.19\right)$ of death obsession variance. See table 2 for these result.

In order to respond to the fifth research question which is about difference of death obsession between women and men, independent $t$ test was utilized that its results have come in table four. The results show that death obsession and its aspects between two groups has significant differences in $\mathrm{p}<0 / 001$ level.As the results of table four shows with comparing means, generally we can say that death obsession with $(M=33 / 73)$ in women is more than men and this discrepancy is meaningful $(\mathrm{p}<0 / 001, \mathrm{df}=428$ and $\mathrm{t}=-5 / 38)$. In aspects of death obsession in death rumination, the mean of women scores with $(M=16 / 69)$ is more than the mean of men scores with $(\mathrm{M}=$ $13 / 94)$ and with $(\mathrm{p}<0 / 001, t=-4 / 63)$ this difference is meaningful. In the aspect of death dominance also mean of women $(M=11 / 04)$ is more than the mean of men with $(M=9 / 30)$ and with $(p<0 / 001, d f=482, t=-5 / 29)$ this difference is meaningful. In the aspect of death idea repetition, the means of girls $(M=5 / 99)$ is more than the mean of boys $(M=4 / 92)$ and with $(p<0 / 001, d f=482, t=4 / 46)$ this difference is meaningful. See table 3 for these results.

\section{Discussion}

The aim of this study was to survey and analyze the relationship between intrinsic and extrinsic religiosity orientation and personality traits with death obsession. The findings indicate that death obsession has more relationship with extrinsic religiosity orientation than intrinsic religiosity orientation. This findings are in agreement with previous researches by Multby and day (2000), Abdel-khalek (2002), Abdel-khalek and lester (2003), Abdel-khale and Al-sabwah (2006), Falkenhain and Handal (2003) and Wink and Scoott (2005). They had reached this conclusion that an intrinsic religious belief in contrast to extrinsic religiosity has less relationship than death with fear and mental health. Most of the studies show that the higher score of intrinsic religiosity the more death obsession decreases. 
Regarding death obsession with extrinsic religiosity orientation can indicate the abortive religiosity of individuals with preoccupation with death. Allport believes that people who their religious activities are external, use religious as a tool for their individual or social goals. Indeed we can make a relationship between death anxiety and conflict believes about death and afterlife and lack of personal philosophy of death with extrinsic religiosity orientation. Researches show that people who have moderate religiosity orientation in comparison to religious ones or persons with low religiosity orientation, fears more than them. From perspective of religiosity, individuals who are not eager so much or unstable about religiosity commitments have more doubts and hesitations about afterlife, punishment or reward after death and without hope for redemption possibly cause more fear for them from death. From a psychological perspective, the lack of a good philosophy of death,

Result in a vulnerable sense of one and it can be an index for chronic anxiety. From a psychological perspective, people with extrinsic religiosity orientation, because of disaffiliation and connection with social institutes, are more likely to norm rupture (social instability). And this makes them more prone to psychotic. Believe that a major factor in people's concerns with extrinsic religiosity orientation about phenomena such as death is believed in afterlife (Wink \& Scott, 2005, Falkenhain \& Handal, 2003).

Macintash and wertman (1993) express that religious people have mental religiosity traps that can help them in cognitive processing about death. It can be deduced that these mental traps can effect individual's assessment of life philosophy and death. And can even control the anxiety caused by thinking about death. Religious people with intrinsic orientation have mental traps that influence their appraisal from stressful events so that these individuals consider the events less stressful, hence less worry and preoccupation with death.

On the other hand in some of the studies, concerns and fear of death demonstrate a relationship with existential well-being and spiritual well-being. Introspection approach, in structural similar to mental scanning, consider the origins of anxiety affairs such as ontological, their existence needs and final concerns forming existence dynamic psychotherapy, such as death loneliness and meaningless that calls anxiety. In terms of both systems, mental analysis and existential of anxiety caused formation of trauma, in this view, anxiety or fear of death, perhaps due to existential awareness of nullity (Weems et al, 2005).

One of the other findings of this research was the relationship between neurticism of five factor personality dimensions and death. That is match with findings of Maltby and Day (2000). They have also reported a positive significant correlation between the Eysenk neuroses with death obsession, such as broad dimensions of personality, stability, emotional stability in the face of emotional instability. Having negative feelings and emotions such as fear, sadness, arousal, anger and guilt forms the basis of emotional instability. The person who is emotionally unstable is more likely to have irrational beliefs and have less power to control the impulses and show weaker degree of compliance with the others and environment. On the other hand the research findings showed that between the five factor personality dimensions except neuroticim, other personality dimensions agreeableness, conscientiousness, extroversion and openness has a significant correlation with intrinsic and extrinsic religiosity orientation, that the most relationship was between conscientiousness with intrinsic religiosity orientation. That the result of research is consistent with the findings of Francis and Bourk (2003), Francis and Pearson (2001), Egan et al. (2004) Henningsgard and Arnau (2008), Saroglou (2002).

The explanation for this finding could be argued, people who have high scores on indicators of neuroticism, emotionally considered as instable individuals; they are usually uneasy, have volatile mood and cannot easily face with difficult situations. Therefore the probability of death obsession and anxiety about this issue may be more in them. On the other hand since extrinsic religiosity orientation is dominant in these individuals, they are not so eager to religious commitments. Religion as a shield has not efficiency against personal anomy (instability); therefore, doubt about afterlife and anxiety about death may occur more in this people.

Another finding of this research was the difference between boys and girls in relation to death obsession. The results showed that death obsession was more in girls than boys. The findings were in complete agreement with findings of, Abdel-khalek (2006, 2007), Abdel-khalek and Lester (2003).

To explain the above findings, high death obsession in female group with death anxiety and some of the fears that probably are more in women than men could be argued. It seems that fear of loneliness, fear of losing family, fear of pain, mostly attention to physical issues among women are more than men. And since they are emotionally not steadier and more stimulant than men may react to anxiety affairs such as death with higher level than men. On the other hand the question of death obsession questionnaire can act as emotional stimuli. Because of sexual a characteristic which exists in men, maybe they don't act realistic in expressing their feels, anxieties and fears against women and show resistance in response to stimulating questions about death. But women act more realistic in expressing their feels, anxieties and fears, and this makes their scores of death obsession be 
more than men. On the other hand based on associate believe network theory, the probability of death stimulating words in death questionnaire as the causes activation of semantic networks in mind of women that is more than men. It seems that the domination of right hemisphere and emotional hemisphere of brain in women can justify these significant nodes. This difference can be justified in creation and cognitive processing of emotional information field and its difference in both male and female. The previous findings have shown high of depression among women than men.

Finally we can claim that religion is able to provide a psychological system for the individuals which can lead him or her toward a stable emotion by strengthening the power of continence. Also, when the beliefs and religiosity to be placed in psychological structure of the person can at least guarantee unity and provide mental health for them. More studies required to be done in Iran culture and religion to reach better and more accurate results. The way of individual's evaluation from threatening factors, self-emotional-control in dealing with situation by semantic ways and impact on the consequence of stressful factors by particular interpretation that religion leaves for the individuals. This is a way to end the apprehension and anxiety or inner turmoil. A particular interpretation that culture and religion puts for individuals, affect his or her cognitive processing of emotional information. The death-obsessed character is mediated by religious orientation (intrinsic or extrinsic) personality traits (extroversion, responsibility, openness to experience, consistency and neurosis) effects religiosity orientation and that effects the perception emotional processing, attention, interpretation, recall and recognition of the concept of death.

Research statistical society and way of research, brings some limitations in generalizing the findings, interpretation and the cause cognitive variables that should be considered. The sample which was examined in this study was a student sample so should be cautious in generalizing the findings to other populations. To examine more accurately the relationship between religiosity orientation and personality traits with death obsession it is proposed that in clinical samples, including samples of patients with OCD (obsessive compulsive disorder), causes be considered. Also recommended in different age during adolescence, youth, middle-aged and elderly (old age), attitudes about death and consequently death obsession be reviewed.

\section{References}

Abdel- khalek, A.M. (1998a). The structure and measurement of death obsession. Personality Indiv. Diff, 24, 159-165.

Addel- khalek, A. M. (1998b). The development and validation of the Arabic obsessive Compulsive scale. European Journal of psychological Assessment, 14, 146-158.

Addel- khalek, A. M. (2002). Death obsession in Egyptian samples: Differences amang people with anxiety disorders, schizophrenia, addictions, and normal's. Death studies, 26, 405-416.

Abdel-Khalek, A. M., \& Lester, D. (2003). Death obsession in Kuwaiti and American college students. Death studies, 27, 541-553.

Adel-Khalek, A. M. Al- Arja, N.S., \& Abdella, T. (2006). Death obsession in Palestinians. Death studies, 30, 203-215.

Al-Sabwah, M. N., \& Abdel-khalek, A. M. (2006). Religiosity and death distress in Arabic college students. Death studies, 30, 365-375.

Abdel- Khalek, A. M. (2007). Love of life and death Distress: Two Spearate factors. Omega, the Journal of Death and Dying, 55, 267-278.

Costa, P. T. R., \& McCrae, R. R. (1986). Personality in adulthood: A six year longitudinal study of self-reports and spouse ratings on the NEO personality Inventory. Journal of personality and Social psychology, 54, 853-863.

Compton, C. (2005). An introduction in positive psychology. USA, Wadsworth Publishing.

Duff, R. W., \& Lawrence, K. (1995). Age density, religiosity and death anexity in retirement communities. Review of religious Research, 37(1), 19-32.

Egan, E., Kroll, J., Carey, K., Johnson, M., \& Erickson, P. (2004). Eysenck personality Scales and religiosity in a use outpatient sample. Personality and Individual differences, 37, 1023-1031.

Francis, L. J., \& Perason, P. R. (2001). Extraversion and religiosity. Journal of social psychology, 125, 269-270.

Francis, L. J., \& Bourke, R. (2003). Personality and religion: applying cattell's model among secondary school pupils. current psychology, 22, 125-137. 
Falkenhain, M., \& Handal, P. J. (2003). Religion, death, attitudes, and belief in afterlife in the elderly. Untangling the relationship, 42, 67-76.

Han S., Qin, Y., \& Ma, Y. (2010). Neurocognitive processes of linguistic cues related to death. Neuropsycholigia, 48, 3436-3442.

Henningsgard, Y. M., \& Arnau, R. C. (2008). Relationships between religiosity and personality and personality: a multivariate analysis. Personality and individual Differences, 42, 703-708.

Hamid, N. (2009). Study the relationships between religious attitudes, mental health and immune system in medical students. Quarterly journal of psychological studies, 5(2), 73-88.

Jorm, A. F., \& Christansem, H. (2004). Religiosity and personality: eridence for nonlinear associations. Personality and Individual Differencess, 36, 1433-1441.

Lonetto, R., \& Templer, D. I. (1986). Death anxiety. Washington, DC:Hemisphere Publishing.

Maltby, Y. (1999). Personality Dimensions of religious orientation. Journal of Personality, 133, 631-640.

Maltby, Y., \& Day, L. (2000). Religious orientation and death obsession. The Journal of Genetic psychology, 16, 122-124.

Maltby, Y., \& Day, L. (2000). The reliability and validity of the death obsession scale among English university and adult samples. Personality and Individual Differences, 28, 695-700.

Rajabi, G. (2009). The psychometric properties of death obsession scale in freshman undergraduate students. Journal of Applied Sciences, 9, 360-365.

Rossenblat, A., Greenberg, Y., Solomon, S., Pyszczynski, T., \& Lyon, D. (1989). Evidence for terror management theory: I. the effects of mortality salience on reactions to those who violate or uphold cultural values. J Pers Socia Psychol, 57, 681-690.

Saroglou, V. (2002). Religion and the Five Factors of personality: a meta-analytic review. Personality and Individual Differences, 32, 15-25.

Sehmeichel, B., Gailliot, M. T., Filardo, E., McGregor, L., \& Gitter, S. (2009). Terror management theory and self-esteem revisited: the roles of implicit and explicit self-esteem in mortality salience effects. Journal of personality and Social Psychology, 69, 1077-1087.

Watson, P. J., Ghorbani, N., G Haramaleki, A., Morrise, R. J., \& Hood, Jr. R. W. (2000). Muslim attiudes toward religion scale:factors,validity and complexity of relationships with mental health in Iran. Mental Health, Religion and Culture, 3(2), 125-132.

Weems, C. F., Costa, N. M., Dehon, C., \& Berman, S. (2004). Paul Tillich theory of existential anxiety: a preliminary conceptual and empirical examination. Anxiety, Stress and Coping, 17(4), 383-399.

Wink, P., \& Scott, J. (2005). Does religiousness buffer against the fear of death and dying in late adulthood? Findings From a longitudinal study. Journal of Gerontology, 60, 207-214. 
Table 1. Means, standard deviations, and correlations among the study variables

\begin{tabular}{|c|c|c|c|c|c|c|c|c|c|c|c|c|c|}
\hline Variable & M & SD & 1 & 2 & 3 & 4 & 5 & 6 & 7 & 8 & 9 & 10 & 11 \\
\hline Death obsession & $31 / 11$ & $1 / 16$ & 1 & & & & & & & & & & \\
\hline Death rumination & $15 / 39$ & $6 / 66$ & $0 / 95^{* *}$ & 1 & & & & & & & & & \\
\hline Death domination & $10 / 22$ & $3 / 71$ & $0 / 84 * *$ & $0 / 69^{* *}$ & 1 & & & & & & & & \\
\hline $\begin{array}{ll}\text { Repeated } & \text { ideas } \\
\text { toward death } & \end{array}$ & $5 / 49$ & $2 / 68$ & $0 / 81 * *$ & $0 / 70^{* *}$ & $0 / 57 * *$ & 1 & & & & & & & \\
\hline $\begin{array}{l}\text { Intrinsic religiosity } \\
\text { Orientation }\end{array}$ & $20 / 63$ & $6 / 31$ & $-0 / 05$ & $-0 / 03$ & $-0 / 04$ & $0 / 06$ & 1 & & & & & & \\
\hline $\begin{array}{l}\text { Extrinsic Religiosity } \\
\text { orientation }\end{array}$ & $28 / 09$ & $5 / 86$ & $0 / 12 * *$ & $0 / 13^{* *}$ & $0 / 04$ & $0 / 13$ & $-0 / 25^{* *}$ & 1 & & & & & \\
\hline Neuroticism & $33 / 47$ & $8 / 39$ & $0 / 42 * *$ & $0 / 36^{* *}$ & $0 / 42 * *$ & $0 / 36^{* *}$ & $0 / 02$ & $0 / 02$ & 1 & & & & \\
\hline Extroversion & $30 / 90$ & $5 / 93$ & $-0 / 15^{* *}$ & $-0 / 15^{* *}$ & $-0 / 12 * *$ & $-0 / 10^{*}$ & $-0 / 23^{* *}$ & $0 / 09^{*}$ & $-0 / 43 * *$ & 1 & & & \\
\hline Openness & $25 / 34$ & $4 / 42$ & $-0 / 02$ & $-0 / 04$ & $-0 / 04$ & $-0 / 04$ & $-0 / 07$ & $-0 / 18^{* *}$ & $-0 / 07$ & $0 / 07^{*}$ & 1 & & \\
\hline Agreeableness & $30 / 05$ & $5 / 43$ & $-0 / 22 * *$ & $-0 / 19 * *$ & $-0 / 21 * *$ & $-0 / 20 * *$ & $-0 / 16^{* *}$ & $-0 / 009$ & $-0 / 42 * *$ & $0 / 29 * *$ & $-0 / 06$ & 1 & \\
\hline Conscientiousness & $36 / 02$ & $6 / 05$ & $-0 / 17 * *$ & $-0 / 17 * *$ & $-0 / 12^{* *}$ & $-0 / 18^{* *}$ & $-0 / 34 * *$ & $-0 / 01$ & $-0 / 28 * *$ & $0 / 48 * *$ & $0 / 09^{*}$ & $0 / 44 * *$ & 1 \\
\hline
\end{tabular}

significant at $\mathrm{p}<.01$, significant at $\mathrm{p}<.05$

Table 2. Summary of step by step regression model for prediction of death obsession through religiosity and personality traits

\begin{tabular}{|c|c|c|c|c|c|}
\hline \multicolumn{6}{|c|}{ Indicator } \\
\hline First step & B & SEB & B & $\mathrm{t}$ & $P$ \\
\hline Neuroticism $\left(\mathrm{R}^{2}=0 / 18\right)$ & 0.58 & 0.057 & 0.424 & 10.27 & $0 / 000$ \\
\hline Second step & & & & & \\
\hline $\begin{array}{r}\text { Neuroticism and extrinsic } \\
\text { religiosity orientation }\left(\mathrm{R}^{2}=0 / 19\right)\end{array}$ & 0.20 & 0.082 & $0 / 10$ & 2.53 & $0 / 01$ \\
\hline
\end{tabular}

Table 3. Statistical index and Independent t Test result for comparison of two groups of boys and girls students' scores in death obsession

\begin{tabular}{|l|ll|ll|lll|}
\hline \multirow{2}{*}{\multicolumn{1}{|c|}{ Variable }} & \multicolumn{3}{c|}{ Girl(n=246) } & \multicolumn{3}{c|}{ Boy(n=238) } & \multicolumn{3}{c|}{} \\
\cline { 2 - 8 } & $\mathrm{M}$ & $\mathrm{SD}$ & $\mathrm{M}$ & $\mathrm{SD}$ & $\mathrm{df}$ & $\mathrm{t}$ & $\mathrm{p}$ \\
\hline Death obsession & $33 / 73$ & $11 / 77$ & $28 / 17$ & $10 / 84$ & 482 & $-5 / 38$ & $0 / 000$ \\
Death rumination & $16 / 69$ & $6 / 85$ & $13 / 94$ & $6 / 13$ & 482 & $-4 / 63$ & $0 / 000$ \\
Death Dominance & $11 / 04$ & $3 / 60$ & $9 / 30$ & $3 / 61$ & 482 & $-5 / 29$ & $0 / 000$ \\
Death idea Repetition & $5 / 99$ & $2 / 73$ & $4 / 92$ & $2 / 51$ & 482 & $-4 / 46$ & $0 / 000$ \\
\hline
\end{tabular}

